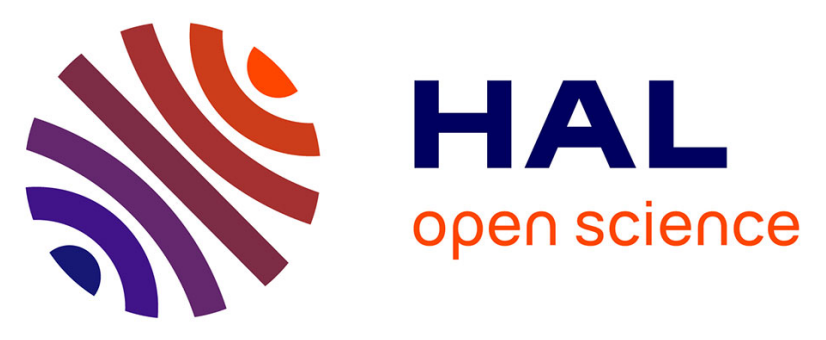

\title{
Direct Measurement of Ionization Charges in Single-phase Liquid Xenon Compton Telescope for $3 \gamma$ Medical Imaging
}

Yajing Xing, Mounir Abaline, Stephane Acounis, Nicolas Beaupere, Jean-Luc Beney, Julien Bert, Stephane Bouvier, Clotilde Canot, Thomas Carlier, Herve Chanal, et al.

\section{To cite this version:}

Yajing Xing, Mounir Abaline, Stephane Acounis, Nicolas Beaupere, Jean-Luc Beney, et al.. Direct Measurement of Ionization Charges in Single-phase Liquid Xenon Compton Telescope for $3 \gamma$ Medical Imaging. 2019 IEEE Nuclear Science Symposium and Medical Imaging Conference (NSS/MIC), Oct 2019, Manchester, United Kingdom. 10.1109/NSS/MIC42101.2019.9059808 . hal-03044155

\section{HAL Id: hal-03044155 \\ https://imt-atlantique.hal.science/hal-03044155}

Submitted on 15 Dec 2020

HAL is a multi-disciplinary open access archive for the deposit and dissemination of scientific research documents, whether they are published or not. The documents may come from teaching and research institutions in France or abroad, or from public or private research centers.
L'archive ouverte pluridisciplinaire HAL, est destinée au dépôt et à la diffusion de documents scientifiques de niveau recherche, publiés ou non, émanant des établissements d'enseignement et de recherche français ou étrangers, des laboratoires publics ou privés. 


\title{
Direct Measurement of Ionization Charges in Single-phase Liquid Xenon Compton Telescope for $3 \gamma$ Medical Imaging
}

\author{
Yajing Xing, Mounir Abaline, Stéphane Acounis, Nicolas Beaupère, Jean-Luc Beney, Julien Bert, Stéphane \\ Bouvier, Clotilde Canot, Thomas Carlier, Hervé Chanal, Michel Cherel, Jean-Pierre Cussonneau, Sara Diglio, \\ Debora Giovagnoli, Jérôme Idier, Françoise Kraeber-Bodéré, Patrick Le Ray, Frédéric Lefèvre, Samuel Manen, \\ Julien Masbou, Eric Morteau, Nicolas Pillet, Didier Roy, Laurent Royer, Jean-Sébastien Stutzmann, Richard \\ Vandaele, Dimitris Visvikis, Yuwei Zhu and Dominique Thers.
}

\begin{abstract}
We report the study of direct measurement of ionization charges in an innovative liquid xenon Compton camera of small animal imaging, named XEMIS2. It was combined with a novel $3 \gamma$ medical imaging modality, showing a "TOF-like" PET performance, targeted to reduce the administered activity diametrically while preserving the image quality in oncology diagnosis. For the Compton cones reconstruction, the sequential low energy electronic recoils from Compton scattering of third $\gamma$ ray $(\sim 1 \mathrm{MeV})$ are measured directly from ionization signals under a high electric field of $2 \mathrm{kV} / \mathrm{cm}$. The Geant4/NEST simulations studies reveal that the intrinsic energy and spatial resolutions of charges carriers measuring in liquid xenon are dominated respectively by the electron-ion recombination and the abstruse trajectory of recoil electrons. Besides, a novel ASIC front-end electronic, XTRACT, and data acquisition chain were developed, dedicated to optimizing the accuracy and efficiency of ionization signals measurement in XEMIS2. It allows a continuous read-out with ultra-low charges threshold and negligible deadtime per individual channel. The performing characteristics were calibrated in the prototype XEMIS1 under the XEMIS2 operation condition, showing a good homogeneous charge linearity response. Meanwhile, the data processing and analysis were developed, where the charge measurement has been optimized through Monte Carlo simulations.
\end{abstract}

\section{INTRODUCTION}

$\mathbf{T}$ HE personalized medicine as the heart of healthcare priorities of the $21^{\text {st }}$ century requires molecular imag-

Manuscript received December 13, 2019. The research presented in this paper has been funded by the E.U., the Region Pays de la Loire in France and by grants from the French National Agency for Research.

Yajing Xing is with SUBATECH, IMT Atlantique, CNRS/IN2P3, Université de Nantes, 44307 Nantes, France (corresponding author e-mail: yajing.xing@subatech.in2p3.fr).

Stéphane Acounis, Nicolas Beaupère, Jean-Luc Beney, Stéphane Bouvier, Clotilde Canot, Jean-Pierre Cussonneau, Sara Diglio, Patrick Le Ray, Frédéric Lefèvre, Julien Masbou, Eric Morteau, Jean-Sébastien Stutzmann, Yuwei Zhu and Dominique Thers are with SUBATECH, IMT Atlantique, CNRS/IN2P3, Université de Nantes, 44307 Nantes, France.

Mounir Abaline, Hervé Chanal, Samuel Manen, Nicolas Pillet, Laurent Royer, Richard Vandaele are with LPC Clermont-Ferrand, 24 Avenue des Landais, Clermont-Ferrand France

Julien Bert, Debora Giovagnoli and Dimitris Visvikis are with INSERM, UMR1101, LaTIM, CHRU Morvan, 29600 Brest, France.

Thomas Carlier and Françoise Kraeber-Bodéré are with Centre Hospitalier Universitaire de Nantes, 44093 Nantes, France.

Michel Cherel is with INSERM U892 équipe 13, 44000 Nantes, France.

Jérôme Idier is with LS2N, Ecole Centrale de Nantes, CNRS/Inp, Université de Nantes, 44307 Nantes, France. ing having better sensitivity and resolution to reduce the administered dose and to shorten the exam time. There are three current evaluations of the Positron Emission Tomography (PET) imaging detectors. The first type is the total-body PET. It reduces the exam time dramatically with the same dose by increasing the field-of-view (FOV), which is highly developed in US [1]. Another approach much explored in the EU is the time-of-flight PET (TOF-PET), whose aim is to decrease the effective length of a line of response (LOR) through accurate time measurement [2]. The third modality aimed at reducing the parallax effects through a depth of interaction on the whole FOV [3]. All those sophisticated detector technologies are based on solid scintillators.

Our team proposed an innovative alternative liquid XEnon Medical Imaging System (XEMIS) [4]. Liquid xenon (LXe), as an emerging monolithic radiation detection medium, has been widely used for particle physics and dark matter detection. It possesses lots of advantageous features, such as high stopping power, ethical sensibility, and simultaneous scintillation light and ionization charge production. Meanwhile, its efficient scalability affords the possibility to develop a total-body imaging detector. Moreover, a "TOF-like" PET performance could be achieve through the application of an innovative $3 \gamma$ imaging modality [5]. It is based on a specific $\left(\beta^{+}, \gamma\right)$ radio-isotope ${ }^{44} \mathrm{Sc}[6]$ and a LXe Compton telescope, measuring with good precision the intersection of LOR and Compton cone to realize a direct 3D location of radio-tracers.

The first R\&D prototype XEMIS1 [7] has been accomplished and showed promising results. A larger Compton camera XEMIS2 [8] for small animal imaging is developed to demonstrate the advantage of liquid xenon ( $\mathrm{LXe}$ ) as a detection medium for $3 \gamma$ imaging. The objective is to obtain a highquality image of small animals with only $20 \mathrm{kBq}$ activity for 20 minutes. The sequential low energy electronic recoils from Compton scattering of third $\gamma-$ ray $(\sim 1 \mathrm{MeV})$ are measured directly from ionization signals under a high electric field of $2 \mathrm{kV} / \mathrm{cm}$. The accurate measurement of ionization charges carriers has thus a vital impact on the Compton cones reconstruction. 


\section{Motivation of ChaRGE MEASUREMENT}

The design of the XEMIS2 Compton camera is shown as Fig. 1. It is composed of two identical single-phase monolithic liquid xenon time projection chambers (LXe-TPC) sharing the same central cathode. Each LXe-TPC has a $12 \mathrm{~cm}$ drift length with the radius varies from $7 \mathrm{~cm}$ to $19 \mathrm{~cm}$. The periphery of the active zone is covered by 64 one-inch PhotoMultiplier tubes to detect the prompting scintillation light. Two-segmented anodes, possessing a total of twenty thousand pixels with a pitch of 3.1 $\mathrm{mm}$, are equipped on both sides to collect the ionization charge carriers.

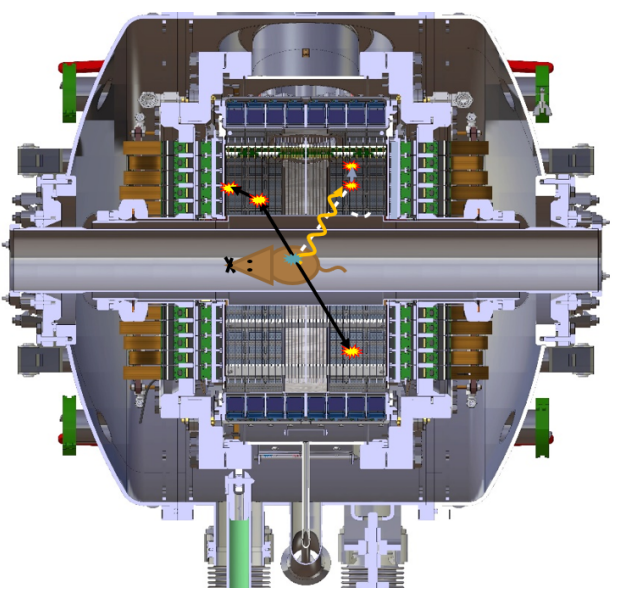

Fig. 1. Design of the XEMIS2 camera for small animal imaging.

In XEMIS2, the scintillation signals mainly provide the $\gamma$ rays interaction time and the spatial pre-localization of $\gamma$-ray interactions to reduce the occupancy of the TPC [9]. The deposited energy and position of each interaction vertex are measured by ionization signals. The ionization charges carriers are directly collected by the segmented anodes which are shielded with Frisch grids, in our single-phase LXe-TPC, without through a gaseous phase electroluminescence amplification.

The accurate measurement of ionization charges carriers plays a vital role in the Compton cones reconstruction, which provides the possibility of Compton interactions sequential analysis and discrimination. Compared with the high cost of PMT/SiPM, the direct ionization signal measurement also has a tremendous economic advantage for the medical imaging application.

\section{STUDY OF ENERGY AND SPATIAL RESOLUTION IN LXE}

The performing energy and spatial resolutions of the XEMIS2 are mainly defined by the intrinsic limits generated by the physic process in LXe and the instrumental measurement method. For example, the spatial resolution of $\mathrm{X}-\mathrm{Y}$ position reconstruction from the instrument is about $100 \mu \mathrm{m}$, which is dominated by the pixel size. In this part, we discussed only the intrinsic limits given by LXe.

The precision of charge carriers measurement in LXeTPC is intrinsically limited by the electrons capture from the electronegative impurities and the electron-ion recombination, leading to attenuation and statistical fluctuation of charge carriers collection.
With our scintillation signal measurement strategy [10], the ionization/scintillation yield anticorrelation will not be applied to correct the recombination influence effect. An intrinsic energy resolution has thus been carried out. In order to optimize the resolution of ionization signals measurement, a high electric field of $2 \mathrm{kV} / \mathrm{cm}$ will be applied in XEMIS2 to increase the ionization yield. Besides, this fluctuation of electrons collection from the electron-ion recombination in LXe can be estimated with NEST [11]. A Geant4/NEST simulation has been done to evaluate this limit, shown in Fig. 2. To eliminate the impact

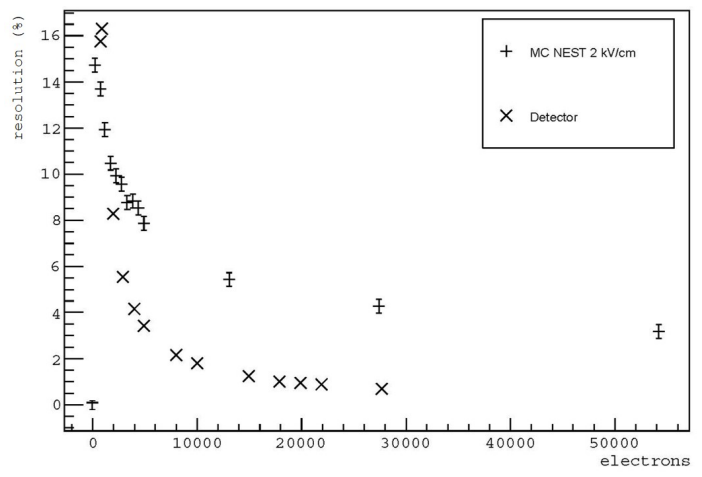

Fig. 2. Intrinsic contribution estimated using NEST comparing with the detector contribution estimated with the simulation of the electronic response. The recombination effect dominates the measurement resolution of large ionization signals (carried charges bigger than 5000 electrons).

of charges attenuation, a very high purity level of xenon is needed since the electronegative impurities dissolved in LXe may absorb the electrons during the drift process. The LXe contamination in the XEMIS1 prototype limited to a ppb level through the purification system [12]. The experimental results demonstrated that the attenuation influence in ultrahigh purity LXe could be neglected before the recombination effect. In XEMIS2, the same/better purity level is expected to be achieved through the new purification-cryogenic subsystem ReStoX [13] equipped a gaseous purification system, realizing a re-circulation closed-loop. The ReStoX and XEMIS2 cryostat are installed in the small animal medical imaging center CIMA at Nantes University Hospital. The current statue is presented in Fig. 3. The intrinsic spatial resolution in LXe is dominated

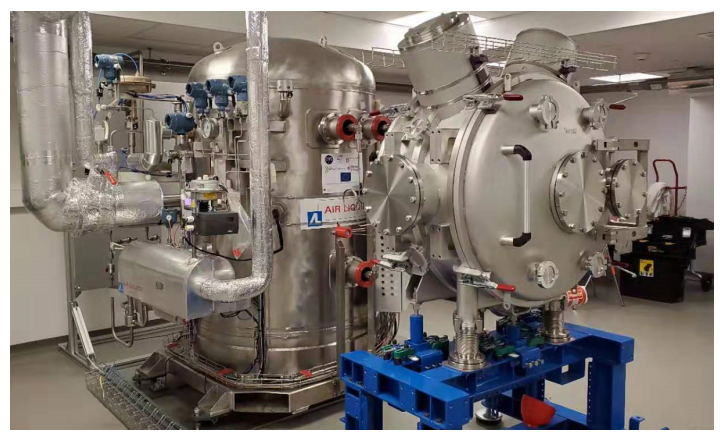

Fig. 3. Installation of the cryogenic subsystem ReStoX and XEMIS 2 cryostat in the small animal medical imaging center CIMA at Nantes University Hospital.

by the distribution of ionized electrons cloud produced from 
electronic recoil. This effect has also been considered and studied by Geant4/NEST simulation, which presents a spatial measurement influence with the energy of electron recoil (about $100 \mu \mathrm{m}$ at $511 \mathrm{keV}$ ).

\section{IONIZATION SIGNALS MEASUREMENT SYSTEM OF XEMIS2}

To achieve this ultra-low activity imaging of small animals, we need to consider three critical issues concerning ionization measurement: resolution optimization, event efficiency maximization and high-frequency signals readout with processable data flow due to low threshold triggering. The published performance calibration of XEMIS1 presented promising results with a roughly $100 \mu \mathrm{m}$ position resolution along the drift coordinate (Z-position) and a good energy resolution of 5\% (9\% FWHM) for $511 \mathrm{keV}$ photoelectric events under an electric field of $1 \mathrm{kV} / \mathrm{cm}[7]$.

To extend the same/better performance from 64 pixels to more than 20000 pixels under a higher electric field of $2 \mathrm{kV} / \mathrm{cm}$ has still several technical hurdles to overcome. Moreover, a considerable quantity of data caused by the ultra-low threshold and self-triggering circuit needs to be managed. For the sake of continuous read-out with negligible dead-time during 20 minutes, the first version of new developed higher capability Data Acquisition (DAQ) chain dedicated to XEMIS2 is accomplished.

\section{A. Front-end electronics and data acquisition chain}

To optimize the event efficiency of XEMIS2 with the negligible dead time, we chose a low threshold self-triggered data acquisition mode for ionization measurement. Besides, in light of the massive number of pixels, the idea of signal information extraction has been brought out to reduce the readout data flow. Based on the above considerations, a novel self-triggered scalable signal readout array with ultra-low charges threshold has been developed in the Subatech laboratory with collaboration.

It consists of four function units: IDeF-X HD-LXe [14], XTRACT, XEMIS Process Unit (XPU) and XEMIS Data Concentrator (XDC). IDeF-x has been validated in XEMIS1, and all the other parts are novel developments, especially XTRACT. It is a multi-channel ASIC $(\times 32)$ with analog and common digital blocks. Each channel includes a Constant Fraction Discrimination (CFD) block which performs the selftriggering function with a limited time walk and an improved timing resolution. XTRACT memorizes only pixel address, amplitude and CFD time of each over-threshold ionization signal. Then, each XPU could connect a maximum of 16 XTRACTs realizing data collection and analog-to-digital conversion. The XDC consists of a Field-Programmable Gate Array (FPGA) component, whose fundamental function is to output the binary data and to synchronize ionization and scintillation DAQ chain. A real-time data filter is also proposed to be implemented in further development. XEMIS2 equipped 640 IDeF-Xs and XTRACTs, 64 XPUs and two FPGAs to read out all ionization signals of 20000 pixels with negligible dead time. This electronic chain is currently tested in XEMIS1 under the XEMIS2 operation condition for precise characterization before integration in XEMIS2.

\section{B. Performing characterization of charge measurement}

The performing characteristics of the recent version of the novel ASIC/DAQ chain were calibrated in the prototype XEMIS1 under the XEMIS2 operation condition. The experimental results have shown that all tested 64 channels of two XTRACTs had a good charge linearity response and quite homogeneous behavior, as presented in Fig. 4. The electronic noise at the DAQ level of each channel is quite uniform and low (about 150 electrons) at the first attempt. Besides, the electronic time resolution and the CFD time walk have also been measured. The DAQ intrinsic time measurement RMS is less than $10 \mathrm{~ns}$, which is limited by FPGA sampling at $100 \mathrm{MHz}$. Besides, results showed that walk correction on the small measured charge is needed. All 800 XTRACTs have been

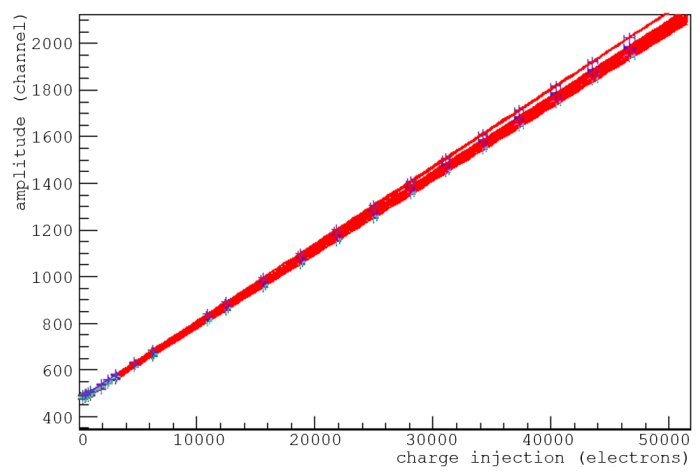

Fig. 4. Experimental charge linearity response of 64 channels of two XTRACTs under XEMIS2 operation condition.

fabricated and the current bench test at room temperature shows a high rate of qualified to $99 \%$.

\section{DATA PROCESSING AND CHARGE MEASUREMENT OPTIMIZATION}

An ultra-low threshold used for self-triggering has been applied to optimize the charge measurement. Hence, the raw data contents abundant over-threshold noise signals during the acquisition. To realize the final interaction vertex reconstruction, we developed a dedicated data processing. After binary data, the electronic calibration results were firstly applied for the sake of pedestal subtraction, gain application and CFD time walk correction. Then, an algorithm has been programmed on soft to realize a data filter in time, reducing the raw data by a factor of 80 (most of the time-isolated noise signals with few ultra-low under-threshold physic signals). We attempt to achieve a real-time data filter in the further R\&D step of XDC. Finally, the practiced spatial Clustering is reconstructed with a time window of $2 \mu \mathrm{s}$, defined by time resolution and time window studies.

A specific detector response Monte Carlo simulation has been done to improve the charge measurement accuracy, concerning the clustering method optimization and Compton sequence reconstruction. The results showed that the ionization signal measurement resolution could be degraded by the mix of two interactions, due to the ionized electrons cloud size, pixel size and electronic integration time. A further finer study 
is under going. Besides, we found that the threshold effect of clustering resulted in a charge loss, presenting a dependence on charge collected number and $\mathrm{z}$ position. A simulation result is shown in Fig. 5, who has been appliqued during the data analysis.

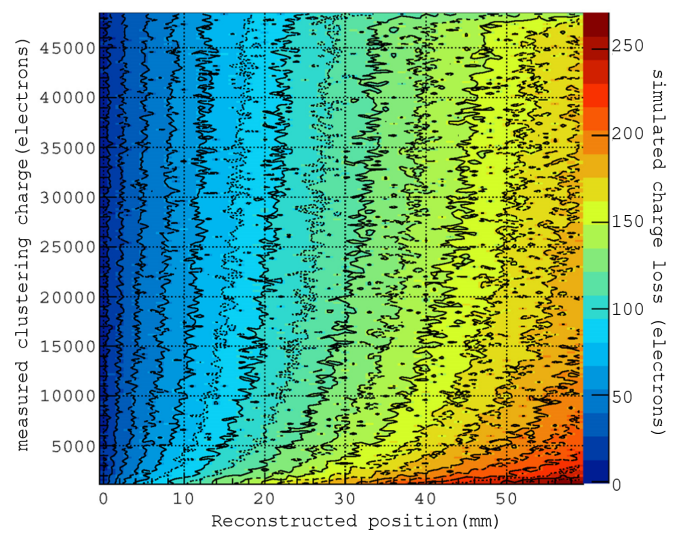

Fig. 5. Mean charge loss with clustering charge and $\mathrm{z}$ position estimated by simulation under the conditions: considering the effects of electron-ion recombination and charge diffusion in LXe; simulating electronics response; neglecting the recoil electron trajectory; under an homogeneous electric field of $2 \mathrm{kV} / \mathrm{cm}$; neglected attenuation influence; equivalent noise charge per pixel equal to 150 electrons.

\section{CONCLUSION}

The accurate Compton scattering reconstruction is a core issue for the success of $3 \gamma$ imaging in XEMIS2. To estimate the ultimate performance, we explored the intrinsic performances of LXe-TPC with Geant4/NEST simulations. Besides, a novel low threshold self-triggered ionization signals measurement system dedicated to XEMIS2 has been currently accomplished and tested in XEMIS1 who presents a good linearity charges response at LXe temperature. All 800 XTRACTs have been fabricated and the first bench test at room temperature shows a high rate of qualified to $99 \%$. Meanwhile, the associated data processing has also been developed. Meanwhile, the data processing and analysis were developed, where charge measurement has been optimized through Monte Carlo simulations. A new approach to Compton scattering angular calibration is underway for further study.

\section{REFERENCES}

[1] R. D. Badawi et al., "First human imaging studies with the EXPLORER total-body PET scanner," Journal of Nuclear Medicine, vol. 60, no. 3, 2019, pp. 299-303.

[2] P. Lecoq, "Pushing the Limits in Time-of-Flight PET Imaging," IEEE Trans. Rad. Plasma Med. Sc., vol. 1, no. 6, Nov. 2017.

[3] E. Yoshida et al., "Development of a whole-body dual ring openpet for inbeam pet," IEEE Transactions on Radiation and Plasma Medical Sciences, 2017, vol. 1, no. 4, p. 293-300.

[4] C. Grignon et al., "Nuclear medical imaging using $\beta+\gamma$ coincidences from Sc-44 radio-nuclide with liquid xenon as detector medium," Nucl. Instrum. Meth. A., vol. 571, 2007, pp. 142-145.

[5] J. P. Cussonneau et al., "3 $\gamma$ Medical Imaging with a Liquid Xenon Compton Camera and 44Sc Radionuclide," Acta Phys. Pol. B, vol. 48, no. 10, pp. 1661, Oct. 2017.
[6] S. Huclier-Markai et al., "Promising Scandium Radionuclides for Nuclear Medicine: A Review on the Production and Chemistry up to In Vivo Proofs of Concept," Cancer biotherapy \& radiopharmaceuticals, vol. 33, no. 8, 2018, pp. 316-329.

[7] L. Gallego Manzano, et al., "XEMIS: A liquid xenon detector for medical imaging," Nucl. Instrum. Meth. A., vol. 787, 2015, p. 89-93.

[8] L. Gallego Manzano et al., "XEMIS2: A liquid xenon detector for small animal medical imaging," Nucl. Instrum. Meth. A., vol. 912, 2018, pp. 329-332.

[9] Y. Zhu et al., "XEMIS2: A liquid xenon Compton camera to image small animals," 2019 IEEE 20th International Conference on Dielectric Liquids (ICDL), Roma, Italy, 2019, pp. 1-4.

[10] Y. Zhu et al., "Scintillation Signal in XEMIS2, a Liquid Xenon Compton Camera with $3 \gamma$ Imaging Technique," 4th International Conference on Technology and Instrumentation in Particle Physics (TIPP), Springer Proc. Phys., vol. 213, 2017, pp. 159-163.

[11] M. Szydagis et al., "NEST: A Comprehensive Model for Scintillation Yield in Liquid Xenon,” JINST, vol. 6, no. 10, 2011, pp. P10002.

[12] Y. Xing et al., "XEMIS: Liquid Xenon Compton Camera for $3 \gamma$ Imaging," 4th International Conference on Technology and Instrumentation in Particle Physics (TIPP), Springer Proc. Phys., vol. 213, 2017, pp. 154-158.

[13] L. Virone et al., "Gravity assisted recovery of liquid xenon at large mass flow rates," Nucl. Instrum. Meth. A., vol. 893, 2018, pp. 10-14.

[14] O. Lemaire et al., "Development of a Readout Electronic for the Measurement of Ionization in Liquid Xenon Compton Telescope Containing Micro-patterns," 2012 IEEE Nuclear Science Symposium Conference (NSS/MIC), Anaheim, CA, 2012, pp. 858-861. 